\title{
JOINT ANGLE-FREQUENCY ESTIMATION USING MULTI-RESOLUTION ESPRIT
}

\author{
Aweke N. Lemma ${ }^{1}$ Alle-Jan van der Veen and Ed F. Deprettere \\ Delft University of Technology, Dept. of Electrical Eng., Delft, The Netherlands
}

\begin{abstract}
A.BSTRACT
Multi-resolution ESPFIT is an extension of the ESPRIT direction finding algorithm to antenna arrays with multiple baselines. A short (half wavelength) baseline is necessary to avoid aliasing, a longr baseline is preferred for accuracy. The MR-ESPRIT algorithm allows to combine both estimates. The same algorithm can be used for multi-resolution frequency estimation, by combining two different sampling frequencies. We show bow this can be used to construct a joint angle-frequency estimator.
\end{abstract}

\section{INTRODUCTION}

Since its derivation in 1983, the ESPRIT algorithm [1] has been used for direction-of-arrival estimation, harmonic analysis, frequency estimation, delay estimation, and combinations thereof. In esisence, the algorithm makes use of a single shift invariance structure present in the array response vector $a(\theta)$, where $\theta=e^{j \mu}$, and $\mu$ is a phase shift to be estimated. In nerrrowband direction-of-arrival estimation, the phase shift is due to the difference in arrival times of the wavefront at the elements of an antenna array. For a uniform linear array (ULA), it is well known that $a(\theta)=\left[\begin{array}{llll}1 & \theta & \theta^{2} & \cdots\end{array}\right]^{T}$ and $\mu=2 \pi \Delta \sin (\alpha)$, where $\Delta$ is the distance between the elements (in wavelengths), and $\alpha$ is the angle of arrival measiured with respect to the normal of the array axis. A similar situation occurs in frequency estirnation where we have $\mu=-2 \pi f T$. Here, $T$ is the sampling period and $f$ is the frequency to be estimated.

In the above simple case (estimation of a single parameter), the accuracy of the estimate of $\sin (\alpha)$ or $f$ is directly proportional to $\frac{1}{2}$ : and $\frac{1}{T}$, respectively. Thus, it is preferable to have a larje baseline $\Delta$ or a large sampling period $T$, so that we collect a large phase shift $\mu$. Unfortunately, however, we cannot collect more than a single cycle, $-\pi \leq \mu<\pi$, because the inverse of the mapping $\mu \rightarrow \theta=e^{\widehat{j \mu}}$ is ambigucius outside this range. To prevent aliasing, we thus have to ensure that $\Delta \leq \frac{1}{2}$ wavelengths, or $T \leq \frac{1}{2} f_{\max }$, which is essentially Shannon's theorem in space and time.

The idea behind multi-resolution parameter estimation is to obtain two or more esitimates of $\mu$ : the first based on a small baseline or short sampling period, yielding a coarse estimate $\mu_{1}$ of $\mu$ without aliasing, and the second based on a large baseline or much larger sampling period, providing an aliased estimate $\mu_{2}$ of $\mu$ at a finer scale. Both estimates can be combined to obtain a final estimate $\hat{\mu}=2 \pi n+\mu_{2}$, where the integer number of cycles $n$ is estimated from $\mu_{1}$.

\footnotetext{
${ }^{1}$ The research of A.N. Lemma is supported by TNO-FEL, The Hague, The Netherlands.
}

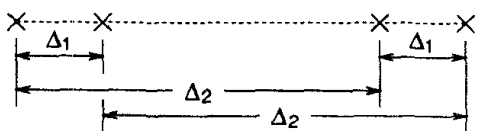

Fig. 1. Multi-resolution spatial sampling

In this paper, we elaborate on this idea, and apply it to DOA estimation, frequency estimation, and a combination of the two. It should be remarked that none of the ideas is truly new. In particular, Zoltowski e.a. [2] discuss a similar problem of angle-frequency estimation using multiple scales in time and space. Because of ambitious goals, however, their solutions are very much directed by engineering considerations, which incurs a certain sacrifice in elegance and clarity. In particular, the coarse frequency estimation is done by applying ESPRIT to a small set of DFT values around spectral peaks which are determined via peak searching algorithms. The fine frequency estimates and the angle estimates are obtained sequentially and for each estimated coarse frequency independently, which assumes that they are sufficiently unique. Here, we derive a one-shot joint estimation procedure referred to as MR-ESPRIT

There is a connection of MR-ESPRIT to MI-ESPRIT $[3,4]$ as well. MI-ESPRIT also exploits the multiple shiftinvariance structure present in multi-baseline arrays. A distinction is that MI-ESPRIT is formulated in terros of (iterative) subspace fitting, and basically attempts to find more accurate beamforming vectors by considering multiple shift invariances. The original paper [3] did not specifically recognize the fact that also more accurate direction estimates can be found. Results in that direction, as well as a non-iterative algorithm, can be found in [4].

\section{MULTI-RESOLUTION ESPRIT}

The original ESPRIT algorithm is based on arrays with a doublet structure, i.e. consisting of several antenna pairs with the same baseline vectors. The chosen array geometries often admit other pairings with different baselines. For instance, the array structure shown in Fig. 1 combines two spatial sampling rates. The minimal number of antennas to have two baseline vector pairs is four. With more antennas, several interesting configurations are possible.

The $M$-dimensional array response vector $a(\alpha)$ is defined as the response of the $M$-element antenna array to a narrowband signal from a direction $\alpha$. It can be parameterized in several ways. The usual parameterization is in terms of the 'electrical angle' $\theta=e^{j 2 \pi \Delta \sin (\alpha)}$, where $\Delta$ is a reference distance smaller than half a wavelength. In our case of an array with two baselines, we can (redundantly) parameterize the array by two parameters, $\theta_{1}=e^{j 2 \pi \Delta_{1} \sin (\alpha)}$ and $\theta_{2}=e^{j 2 \pi \Delta_{2} \sin (\alpha)}$. In the case of the array of Fig. 1, we 
have

$$
\boldsymbol{a}\left(\theta_{1}, \theta_{2}\right)=\left[\begin{array}{c}
1 \\
\theta_{1} \\
\theta_{2} \\
\theta_{1} \theta_{2}
\end{array}\right]
$$

The idea is to treat the two parameters as independent and estimate both of them from the measurement data, and only then combine them into a single estimate of $\sin (\alpha)$. Estimation is done by exploiting the dual shift-invariance structure of $\boldsymbol{a}\left(\theta_{1}, \theta_{2}\right)$, i.e., in the above example

$$
\begin{aligned}
& a_{x 1}=\left[\begin{array}{l}
a_{1} \\
a_{3}
\end{array}\right], a_{y 1}=\left[\begin{array}{l}
a_{2} \\
a_{4}
\end{array}\right] \quad \Rightarrow \quad a_{y 1}=a_{x 1} \theta_{1}, \\
& a_{x 2}=\left[\begin{array}{l}
a_{1} \\
a_{2}
\end{array}\right], a_{y 2}=\left[\begin{array}{l}
a_{3} \\
a_{4}
\end{array}\right] \quad \Rightarrow \quad a_{y 2}=a_{x 2} \theta_{2},
\end{aligned}
$$

where $a_{i}$ is the $i$-th entry of $a\left(\theta_{1}, \theta_{2}\right)$. For more general arrays with a dual shift-invariance structure, we can define selection matrices $\boldsymbol{J}_{x i}$ and $\boldsymbol{J}_{y i}(i=1,2)$ such that the above relations hold for $\boldsymbol{J}_{x i} \boldsymbol{a}$ and $\boldsymbol{J}_{y_{i}} \boldsymbol{a}$. The resulting ESPRITtype algorithms are very similar to the case of joint azimuthelevation estimation.

Thus, to be specific, consider $d$ narrowband sources $s_{i}(t)$ impinging on the antenna array. Collecting $N$ output samples of the $M$ antenna outputs into an $M \times N$ data matrix $\boldsymbol{X}$ in the usual way, we obtain the data model

$$
X=A S=a_{1} s_{1}+\cdots+a_{d} s_{d}
$$

where the columns of $\boldsymbol{A}$ contain the array response vectors $\left\{a_{i}\right\}$, and the rows of $S$ are the sampled source signals. Assuming $d<M$, the first step of the algorithm is to estimate a basis $\boldsymbol{U}$ of the column span of $\boldsymbol{X}$, typically using an SVD. $\boldsymbol{U}$ and $\boldsymbol{A}$ are related by a $d \times d$ nonsingular matrix $\boldsymbol{T}$ as

$$
\boldsymbol{U}=\boldsymbol{A T}
$$

The second step in the algorithm is to form submatrices of $U$ using the proper selection matrices:

$$
\boldsymbol{U}_{x i}=J_{x i} U, \quad U_{y i}=J_{y i} U . \quad(i=1,2)
$$

The shift-invariance structure of the array implies that

$$
U_{x i}=A^{\prime} T, \quad U_{y i}=A^{\prime} \Theta_{i} T,
$$

where $\boldsymbol{A}^{\prime}$ consists of the first $M-1$ rows of $\boldsymbol{A}$ and the diagonal matrix $\Theta_{i}=\operatorname{diag}\left\{\theta_{i j}\right\}_{j=1}^{d}$ contains the $d$ shift parameters of the $d$ sources with reference to the $i$-th baseline. The final step is to estimate the parameters by considering

$$
\begin{aligned}
& \boldsymbol{E}_{1}=\boldsymbol{U}_{\boldsymbol{T} 1}^{\dagger} \boldsymbol{U}_{y_{1}}=\boldsymbol{T}^{-1} \Theta_{1} \boldsymbol{T}, \\
& \boldsymbol{E}_{2}=\boldsymbol{U}_{\boldsymbol{x} 2}^{\dagger} \boldsymbol{U}_{y_{2}}=\boldsymbol{T}^{-1} \Theta_{2} \boldsymbol{T} .
\end{aligned}
$$

It is seen that the data matrices $\boldsymbol{E}_{1}$ and $\boldsymbol{E}_{2}$ are jointly diagonalizable by the same matrix $\boldsymbol{T}$. There are several algorithms to compute this joint diagonalization, e.g. by means of QZ iterations [5] or Jacobi iterations [6]. For this to work, it is necessary that each submatrix $U_{x i}$ has at least $\boldsymbol{d}$ rows. After $\boldsymbol{T}$ has been found, we also have estimates of $\left\{\left(\theta_{1 k}, \theta_{2 k}\right)\right\}$ for each of the $d$ sources.

It remains, for each source, to combine $\theta_{1}$ and $\theta_{2}$ into an estimate of the argument $\mu$ of $\theta$. Let us assume that $\Delta_{1} \leq \frac{1}{2}$, so that $\mu_{1}$ (argument of $\theta_{1}$ ) is not aliased and is a coarse estimate of $\mu$. Also assume that $\Delta_{2} \gg \frac{1}{2}$, so that in $\mu_{2}$ aliasing occurs: the estimate $\mu$ is proportional to $\mu_{2}$

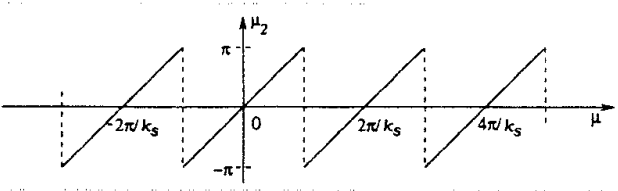

(a)

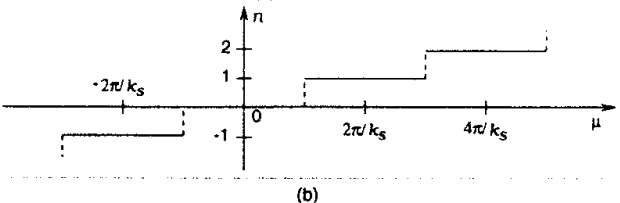

Fig. 2. (a) The aliased spatial frequency $\mu_{2}$ as a function of the alias-free spatial frequency $\mu$.

(b) The corresponding winding number $n$.

plus an appropriate integer multiple of $2 \pi$ (see Fig. 2). It follows that we have two estimates of $2 \pi \sin (\alpha)$,

$$
2 \pi \sin (\alpha)=\frac{1}{\Delta_{1}} \mu_{1}=\frac{1}{\Delta_{2}}\left(2 \pi n+\mu_{2}\right) .
$$

The winding number $n$ is determined as the best fitting integer to match the two right hand side expressions,

$$
n=\operatorname{round} \frac{1}{2 \pi}\left(\frac{\Delta_{2}}{\Delta_{1}} \mu_{1}-\mu_{2}\right) \text {. }
$$

The ratio $k_{s}:=\frac{\Delta_{2}}{\Delta_{1}}$ can be interpreted as the (spatial) gain in resolution. In particular, the estimate of $2 \pi \sin (\alpha)$ based on $\mu_{2}$ is a factor $k_{s}$ more accurate than that based on $\mu_{1}$. Assuming for simplicity that $\mu_{1}$ and $\mu_{2}$ are independent variables with equal variances, the two estimates can be optimally combined as

$$
\sin (\alpha)=\frac{s_{1}+k_{s}^{2} s_{2}}{1+k_{s}^{2}}
$$

$$
\text { where } s_{1}=\frac{1}{2 \pi} \frac{1}{\Delta_{1}} \mu_{1} \quad \text { and } \quad s_{2}=\frac{1}{2 \pi} \frac{1}{\Delta_{2}}\left(2 \pi n+\mu_{2}\right)
$$

(This expression easily generalizes to the case of more than two baselines.)

\section{JOINT ANGLE-FREQUENCY ESTIMATION}

\subsection{Model}

Suppose that we observe a frequency band of interest, and want to separate all sources that are present. The sources are narrow band, typically with different carrier frequencies, but the spectra might be partly overlapping. The objective is to construct a beamformer to separate the sources based on differences in angles or carrier frequencies. This is a problem of joint angle-frequency estimation $[2,7,8]$. We will assume that the sample rates are much higher than the data rates of each source, and that there is no multipath, although generalizations are possible.

Suppose that the narrow band signals have a bandwidth of less than $\frac{1}{T}$, so that they can be sampled with a period $T$ to satisfy the Nyquist rate. We normalize to $T=1$. Let us say that the bandwidth of the band to be scanned is an integer number $F$ times larger: after demodulation to IF we have to sample at a rate $F$. Without multipath, the data model of the modulated sources at the receiver is

$$
\boldsymbol{x}(t)=\sum_{1}^{d} a\left(\theta_{i}\right) e^{j \frac{2 \pi}{F} f_{i} t} s_{i}(t)
$$


where $f_{i}$ is the residuial modulation frequency of the $i$-th source $\left(-\frac{F}{2} \leq f_{i}<\frac{F}{2}\right)$. In matrix form this is written as

$$
\boldsymbol{x}(t)=\boldsymbol{A} \boldsymbol{\Phi}^{t} \boldsymbol{s}(t)
$$

where $\boldsymbol{\Phi}=\operatorname{diag}\left\{\phi_{i}\right\}_{i=1}^{d}$ and $\phi_{i}=e^{j \frac{2 \pi}{F} f_{i}}$. Since $F$ can be quite large (order 20, say), it would be very expensive to construct a full data matrix of all samples. In fact, it is sufficient to subsample: collect $m$ subsequent samples at rate $F$, then wait till the next period before sampling again, resulting in a data matrix

$$
\boldsymbol{X}=\left[\begin{array}{llll}
x(0) & \boldsymbol{x}(1) & \cdots & \boldsymbol{x}(N-1) \\
\boldsymbol{x}\left(\frac{1}{F}\right) & \boldsymbol{x}\left(1+\frac{1}{F}\right) & \cdots & \boldsymbol{x}\left(N-1+\frac{1}{F}\right) \\
\vdots & \vdots & & \vdots \\
x\left(\frac{m-1}{F}\right) & x\left(1+\frac{m-1}{F}\right) & \cdots & x\left(N-1+\frac{m-1}{F}\right)
\end{array}\right]
$$

With the model of $x(t)$ in (2), we find that $X$ has a factorization

$$
\boldsymbol{X}=\left[\begin{array}{lll}
\boldsymbol{A s}(0) & \boldsymbol{A} \boldsymbol{\Phi}^{F} \boldsymbol{s}(1) & \cdots \\
\boldsymbol{A} \boldsymbol{\Phi} \boldsymbol{s}\left(\frac{1}{F}\right) & \boldsymbol{A \Phi}^{F+1} \boldsymbol{s}\left(1+\frac{1}{F}\right) & \cdots \\
\vdots & \vdots & \\
\boldsymbol{A} \boldsymbol{\Phi}^{m-1} \boldsymbol{s}\left(\frac{m-1}{F}\right) & \boldsymbol{A \Phi}^{F+m-1} \boldsymbol{s}\left(1+\frac{m-1}{F}\right) & \cdots
\end{array}\right]
$$

Let us assume at this point that $F \gg m$. In that case, $s(t)$ is relatively band limited with respect to the observed band, which allows to make the crucial assumption that

$$
s(t) \approx s\left(t+\frac{1}{F}\right) \approx \cdots \approx s\left(t+\frac{m-1}{F}\right)
$$

so that the model of $X$ simplifies to

$$
\begin{aligned}
\boldsymbol{X} & \approx\left[\begin{array}{c}
\boldsymbol{A} \\
\boldsymbol{A} \boldsymbol{\Phi} \\
\vdots \\
\boldsymbol{A} \boldsymbol{\Phi}^{m-1}
\end{array}\right]\left[\begin{array}{lllll}
\boldsymbol{s}_{0} & \boldsymbol{\Phi}^{F} \boldsymbol{s}_{1} & \cdots & \boldsymbol{\Phi}^{(N-1) F} \boldsymbol{s}_{N-1}
\end{array}\right] \\
& =(\boldsymbol{F} \circ \boldsymbol{A})\left(\boldsymbol{F}^{\prime} \odot \boldsymbol{S}\right) .
\end{aligned}
$$

where $\diamond$ represents a column-wise Kronecker product, $\odot$ clenotes an entry-wise (Schur-Hadamard) matrix product and

$$
\boldsymbol{F}=\left[\begin{array}{ccc}
1 & \cdots & 1 \\
\phi_{1} & \cdots & \phi_{r} \\
\vdots & & \vdots \\
\phi_{1}^{F-1} & \cdots & \phi_{r}^{F-1}
\end{array}\right], \quad \phi_{i}:=\phi^{f_{i}}=e^{-j \frac{2 \pi}{r} f_{i}} .
$$

$\boldsymbol{F}^{\prime}$ is similar to $\boldsymbol{F}$ except for a transpose and different powers, and represents the modulation on the signals. Obviously, beamforming will not remove this modulation but after estimating $\boldsymbol{\Phi}$, we can easily correct for it.

\subsection{Estimation algorithm}

At this point, we have obtained a model with much the same structure as before, but with $\boldsymbol{A}$ replaced by $\boldsymbol{F} \diamond \boldsymbol{A}$. The construction of the beamformer can now follow the same strategy as well. First note that the rank of $\boldsymbol{X}$ is only $d$, since this is the number of rows of $S$. Thus we compute the SVD of $\boldsymbol{X}$, i.e. $\boldsymbol{X}=: \hat{\boldsymbol{U}} \hat{\boldsymbol{\Sigma}} \hat{\boldsymbol{V}}$ where $\hat{\boldsymbol{U}}$ has $d$ columns, spanning the column space of $\boldsymbol{X}$. As before, we have

$$
\hat{U}=(\boldsymbol{F} \circ \boldsymbol{A}) \boldsymbol{T}, \quad \boldsymbol{S}=\left(\boldsymbol{T} \hat{U}^{H}\right) \boldsymbol{X}
$$

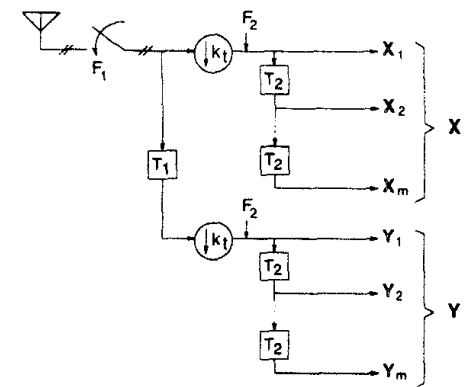

Fig. 3. Multi-resolution temporal sampling

To estimate $\boldsymbol{T}$, we begin by defining two types of selection matrices: a pair to select submatrices from $\boldsymbol{F}$, and a pair to select from $\boldsymbol{A}$,

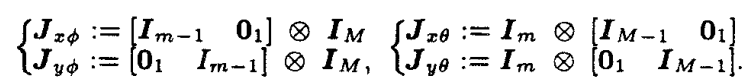

To estimate $\Phi$, we take submatrices consisting of the first and respectively last $M(m-1)$ rows of $\hat{U}$, i.e.

$$
\hat{U}_{x \phi}=J_{x \phi} \hat{U}, \quad \hat{U}_{y \phi}=J_{y \phi} \hat{U},
$$

whereas to estimate $\Theta$ we stack, for all $m$ blocks, its first and respectively last $M-1$ rows:

$$
\hat{U}_{x \theta}=J_{x \theta} \hat{U}, \quad \hat{U}_{y \theta}=J_{y \theta} \hat{U} .
$$

These data matrices have the structures

$$
\left\{\begin{array} { l } 
{ \hat { U } _ { x \phi } = A ^ { \prime } T } \\
{ \hat { U } _ { y \phi } = A ^ { \prime } \Phi T }
\end{array} \quad \left\{\begin{array}{l}
\hat{U}_{x \theta}=A^{\prime \prime} T \\
\hat{U}_{y \theta}=A^{\prime \prime} \Theta T
\end{array},\right.\right.
$$

If dimensions are such that these are low-rank factorizations, then

$$
\begin{aligned}
& \hat{U}_{x \phi}^{\dagger} \hat{U}_{y \phi}=T^{-1} \Phi T \\
& \hat{U}_{x \theta}^{\dagger} \hat{U}_{y \theta}=T^{-1} \Theta T,
\end{aligned}
$$

and $T$ can be computed by joint diagonalization.

\subsection{Multi-resolution estimation algorithm}

If the frequency band to be monitored is much wider than the bandwidth of the signals, then in the above algorithm $F$ has to be chosen very large. This implies that the frequency estimates will not be very accurate. To overcome this problem, we can employ the multi-resolution ideas of section 2, but now in the temporal domain.

Thus suppose we have two sampling rates, $F_{1}$ (fast) and $F_{2}$ (intermediate, compared to the source bandwidths), related via a temporal gain factor $k_{t}=\frac{F_{1}}{F_{2}} \gg 1$. It usually suffices to collect only two subsequent samples at the fastest rate $F_{1}$, for every $m$ samples at the intermediate rate $F_{2}$. See Fig. 3 . In the figure, the outputs $X_{i}$ and $\boldsymbol{Y}_{i}$, $i=1, \ldots, m$, are all $M \times N$ matrices, where $M$ is the number of antenna elements and $N$ is the number of temporal samples. This means that the data matrices $\boldsymbol{X}$ and $\boldsymbol{Y}$ are each of size $m M \times N$ and have a structure as in equation $(3)^{2}$. Let $Z$ be the overall data matrix, with a factorization

$$
z:=\left[\begin{array}{l}
X \\
\boldsymbol{Y}
\end{array}\right]=\left[\begin{array}{l}
\mathcal{A} \\
\mathcal{A} \Phi_{1}
\end{array}\right] S
$$

\footnotetext{
${ }^{2}$ Note that $F_{2}$ corresponds to the sampling rate $F$ in (3).
} 
where $\mathcal{A}$ is constructed from $\boldsymbol{A}$ as

$$
\mathcal{A}=\left[\begin{array}{l}
A \\
A \Phi_{2} \\
\vdots \\
A \Phi_{2}^{m-1}
\end{array}\right] .
$$

The estimation of the parameters follows by defining three types of selection matrices operating on the data matrix $\boldsymbol{Z}$ :

$$
\begin{aligned}
& \boldsymbol{J}_{x \phi 1}=\left[\begin{array}{ll}
1 & 0
\end{array}\right] \otimes \boldsymbol{I}_{m} \otimes \boldsymbol{I}_{M} \\
& \boldsymbol{J}_{y \phi 1}=\left[\begin{array}{ll}
0 & 1
\end{array}\right] \otimes \boldsymbol{I}_{m} \otimes \boldsymbol{I}_{M} \\
& \boldsymbol{J}_{\boldsymbol{x} \boldsymbol{}}=\boldsymbol{I}_{2} \otimes\left[\begin{array}{ll}
\boldsymbol{I}_{m-1} & 0
\end{array}\right] \otimes \boldsymbol{I}_{M} \\
& \boldsymbol{J}_{y \phi 2}=\boldsymbol{I}_{2} \otimes\left[\begin{array}{ll}
\mathbf{0} & \boldsymbol{I}_{m-1}
\end{array}\right] \otimes \boldsymbol{I}_{M} \\
& \boldsymbol{J}_{\boldsymbol{x} \theta}=\boldsymbol{I}_{2} \otimes I_{m} \otimes\left[\begin{array}{ll}
\boldsymbol{I}_{M-1} & 0
\end{array}\right] \\
& \boldsymbol{J}_{y \theta}=\boldsymbol{I}_{2} \otimes \boldsymbol{I}_{m} \otimes\left[\begin{array}{ll}
\mathbf{0} & \boldsymbol{I}_{M-1}
\end{array}\right]
\end{aligned}
$$

After similar processing steps as before (SVD of $Z$, application of the selection matrices), we obtain the model

$$
\begin{aligned}
& U_{x \phi 1}^{\dagger} U_{y \phi 1}=T^{-1} \Phi_{1} T \\
& U_{x \phi 2}^{\dagger} U_{y \phi 2}=T^{-1} \Phi_{2} T \\
& U_{x \theta}^{\dagger} U_{y \theta}=T^{-1} \Theta \boldsymbol{T}
\end{aligned}
$$

A joint diagonalization of the three matrices now provides estimates of $\left(\phi_{1}, \phi_{2}, \theta\right)$ of each source. If the array has an additional multiresolution structure, then $\Theta$ splits into $\Theta_{1}, \Theta_{2}$ and can be estimated with multi-resolution ESPRIT as discussed in section 2.

To complete our estimation procedure, we need to estimate for each source the argument $\mu$ of $\phi$ by combining $\phi_{1}$ and $\phi_{2}$ in the same way as we did for the DOA estimation in section 2 . We assume that the two sampling frequencies $F_{1}$ and $F_{2}$ are such that $F_{1}>\frac{1}{2} f_{\max }$ and $F_{2} \ll \frac{1}{2} f_{\max }$. Based on these two sampling rates we obtain two estimates of the frequency $f$ :

$$
f=\frac{F_{1}}{2 \pi} \mu_{1}=\frac{F_{2}}{2 \pi}\left(2 \pi n+\mu_{2}\right)
$$

The winding number $n$ is determined as before using

$$
n=\operatorname{round} \frac{1}{2 \pi}\left(\frac{F_{1}}{F_{2}} \mu_{1}-\mu_{2}\right)
$$

Note that if $\mu_{1}$ and $\mu_{2}$ have estimation errors of equal size, then the noise on the second estimate is a factor $k_{t}=\frac{F_{1}}{F_{2}}$ smaller than the first estimate. Thus, we would either use the second equation to estimate $f_{i}$, or optimally combine the two estimates using an equation similar to (1).

\section{SIMULATION RESULTS}

In this section we give simple simulation results that demonstrate the theory developed in this paper. The simulation example considers a processing band of $10 \mathrm{MHz}$ and a linear antenna array with $M=4$ antenna elements arranged as in Fig. 1 with $\Delta_{1}=\frac{1}{2}$ and varying $\Delta_{2}$. The data is collected into a $2 m M \times N^{2}$ matrix using the setup of Fig. 3 with $m=2, N=64, F_{1}=20 \mathrm{MHz}$ and varying $F_{2}$. We consider two sources emitting narrowband signals $(25 \mathrm{kHz})$ at center frequencies $f=[6,6.5] \mathrm{MHz}$, and propagating in distinct directions with respective DOAs $\alpha=[40,45]$ degrees. The simulations are intended to indicate the effects of the spatial gain factor $k_{s}$ and the temporal gain factor $k_{t}$. Note that $k_{s}=2$ and $k_{t}=1$ correspond to uniform spatial and temporal sampling, respectively. (a)

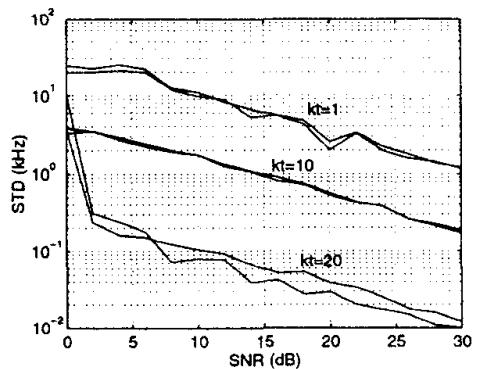

(b)

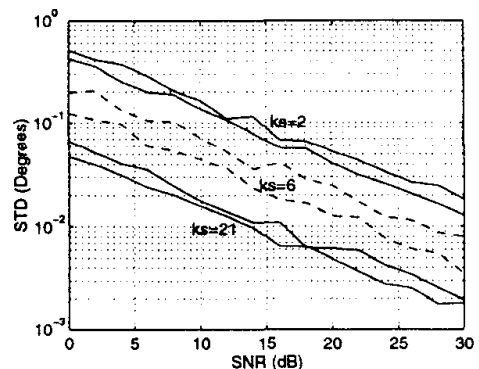

Fig. 4. (a) Standard deviation of the frequency estimates for various temporal gain factors $k_{t}$; (b) similar for the azimuth angle and spatial gain $k_{s}$

The results are plotted in Fig. 4 as functions of varying SNR. From the plots, it is evident that the multi-resolution ESPRIT gives frequency and DOA estimates whose accuracies are proportional to the temporal gain factor $k_{t}$ and the spatial gain factor $k_{s}$, respectively. An upper limit for these gains is reached when the winding numbers $n$ can no longer be estimated accurately; this is dependent on several factors including the SNR.

\section{REFERENCES}

[1] R.H. Roy, ESPRIT-Estimation of Signal Parameters via Rotational Invariance Techniques. $\mathrm{PhD}$ thesis, Stanford University, Stanford, CA, 1987.

[2] M.D. Zoltowski and C.P. Mathews, "Real-time frequency and 2-D angle estimation with subnyquist spatio-temporal sampling," IEEE Trans. Signal Processing, vol. SP-42, pp. 2781-2794, October 1994

[3] A.L. Swindlehurst, B. Ottersten, R. Roy, and T Kailath, "Multiple invariance ESPRIT," IEEE Trans. Signal Processing, vol. 40, pp. 867-881, April 1992.

[4] K.T. Wong and M.D. Zoltowski, "Closed-form multidimensional multi-invariance ESPRIT," in Proc. ICASSP'97, pp. 3489-3493, April 1997.

[5] A.-J. van der Veen and A. Paulraj, "An analytical constant modulus algorithm," IEEE Trans. Signal Processing, vol. 44, pp. 1136-1155, May 1996.

[6] A.-J. van der Veen, P.B. Ober, and E.F. Deperettere, "Azimuth and elevation computation in high resolution DOA estimation," IEEE Trans. Signal Processing, vol. 40, pp. 1828-1832, July 1992.

[7] K.-B. Yu, "Recursive super-resolution algorithm for low-elevation target angle tracking in multipath," IEE Proceedings - Radar, Sonar and Navigation, vol. 141, pp. 223-229, Aug. 1994.

[8] M. Haardt and J. A. Nossek, "3-D unitary ESPRIT for joint 2-D angle and carrier estimation," in Proc. ICASSP'97, pp. 255-258, April 1997. 